\title{
AMEGO: Transients and Multi-Messenger Sources
}

\author{
Judith Racusin* \\ $N A S A / G S F C$ \\ E-mail: judith.racusin@nasa.gov
}

\author{
Amy Lien $^{a}$, Dieter Hartmann ${ }^{b}$ \\ ${ }^{a} U M D / C R E S S T / G S F C$ \\ ${ }^{b}$ Clemson University
}

The AMEGO Team

https://asd.gsfc.nasa.gov/amego/

\begin{abstract}
The All-Sky Medium Energy Gamma-ray Observatory (AMEGO) will survey the entire sky every 3 hours with its wide field of view and excellent continuum sensitivity between $200 \mathrm{keV}$ and $10 \mathrm{GeV}$, discovering new sources and automatically providing follow-up observation for multiwavelength and multi-messenger transient sources. Many of these sources have the energy spectral peaks in the AMEGO band, and/or spectral behavior that is not well understood at these energies. AMEGO will discover new gamma-ray bursts, magnetar flares, active galaxy flares, novae, and monitor the long-term light curves of variable sources including Galactic binaries. Thanks to its wide FoV and survey strategy, AMEGO is also well placed to search for electromagnetic counterparts to gravitational wave and neutrino sources.
\end{abstract}

35th International Cosmic Ray Conference - ICRC2017

10-20 July, 2017

Bexco, Busan, Korea

${ }^{*}$ Speaker. 


\section{AMEGO}

The All-Sky Medium Energy Gamma-ray Observatory (AMEGO) is a mission in consideration as a probe for the 2020 Astrophysics Decadal review. AMEGO will survey the entire sky every 3 hours and see $80 \%$ of the sky every orbit with its wide field of view $(\sim 2.5 \mathrm{sr})$ and excellent continuum sensitivity between $200 \mathrm{keV}$ and $10 \mathrm{GeV}$. The AMEGO design uses primarily well understood, well tested technologies with significant space heritage.

AMEGO will detect medium energy gamma-rays via pair production (between $\sim 10 \mathrm{MeV}$ and $10 \mathrm{GeV}$ ) and via Compton Scattering (between $200 \mathrm{keV}$ and $\sim 10 \mathrm{MeV}$ ). An incoming photon will undergo one of these interactions in a tracker composed of 60 layers of double sided silicon strip detectors coupled to an analogue readout. This tracker will record the energies and tracks of electrons and positrons as they pass through it. A Cadmium Zinc Telluride (CZT) calorimeter is placed under the tracker as well as half way up the sides to measure the location and energy of Compton scattered photons. Finally a CsI calorimeter resides under the lower CZT which extends the upper energy range by measuring the energies of the particles produced via pair production and provides sensitivity to polarization and increased spectral capabilities. The full instrument is surrounded by a plastic anti coincidence detector to veto charge particle backgrounds. Figure 1

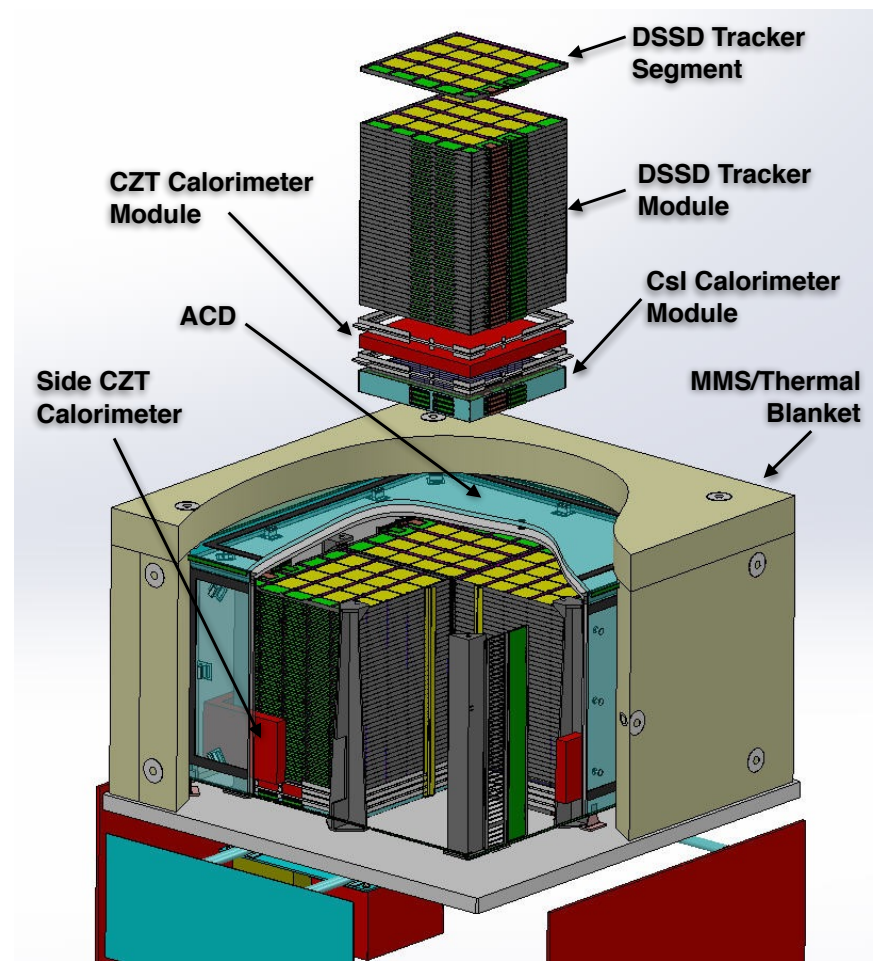

Figure 1: The AMEGO instrument and its subsystems are optimized for continuum sensitivity over a wide field of view and broad medium energy gamma-ray band. 
shows a schematic diagram of the instrument and its subsystems. For more details on the AMEGO mission see $[1,2,3,4,5]$.

Over the past several decades the High Energy (100 MeV to $100 \mathrm{GeV}$ ) sky has been elegantly surveyed by the Fermi Gamma-ray space telescope and several instruments such as HESS, VERITAS, MAGIC, and HAWC have opened up new windows in the Very High Energy (VHE) sky $(\mathrm{E}>100 \mathrm{GeV})$. These missions and experiments have begun a new gamma-ray astronomy and discovered many different types of objects. However, the medium energy gamma-ray sky has not been surveyed sensitively since COMPTEL was flown on CGRO two decades ago. The MeV sky is a difficult region to explore due to the high backgrounds and convergence of two types of detection methods (Compton and Pair) but the rewards are too great to ignore and the technology proven on the Fermi Large Area Telescope (LAT) can be used to build a successful mission.

\section{Science Goals}

The AMEGO energy band bridges the transition between the thermal and non-thermal Universe. It is the only part of the electromagnetic spectrum where it is possible to directly observe nuclear processes (atomic nuclei de-excitations and excitations). Specifically, it covers the positron annihilation line at $511 \mathrm{keV}$. Also, large populations of known sources exist with their peak power output in the $\mathrm{MeV}$ range making it crucial to study for source energetics. The AMEGO mission will initiate breakthroughs in our understanding of extreme environments. The AMEGO science menu focuses on the following topics:

- Astrophysical Jets: Understand the formation, evolution, and acceleration mechanisms in astrophysical jets.

- Compact Objects: Identify the physical processes in the extreme conditions around compact objects.

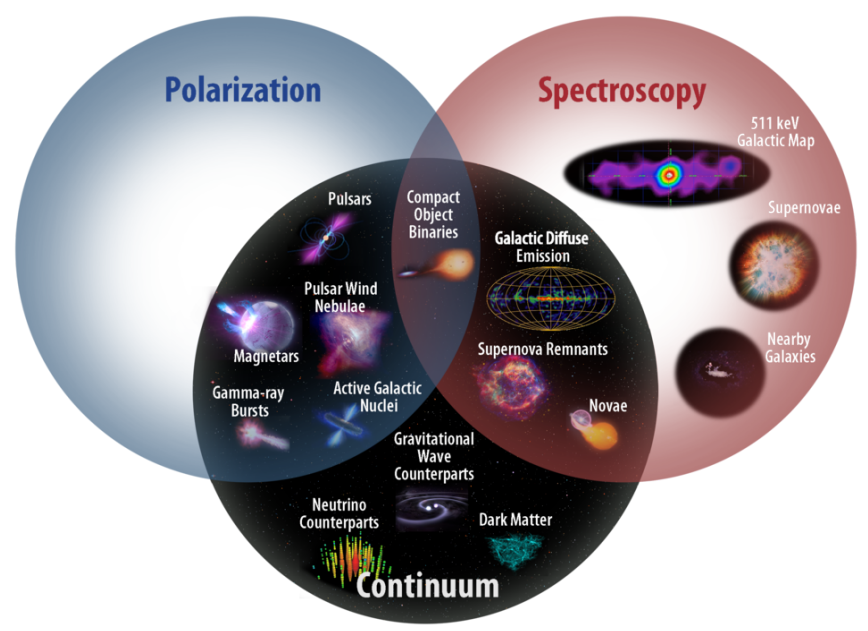

Figure 2: AMEGO is optimized for excellent continuum sensitivity which provides much of the scientific capability, but some source classes will also benefit from MeV spectroscopy and polarization. 
- MeV Spectroscopy: Measure the properties of element formation in dynamic systems.

- Dark Matter: Test models that predict dark matter signals in the MeV band.

These physical properties exist in an array of objects (see Figure 2) including Pulsars and Magnetars, Gamma-ray Bursts and Multi-messenger Astrophysics, Active Galaxies (see Perkins et al., ICRC 2017 proceedings), and Dark Matter (see Caputo et al., ICRC 2017 proceedings).

\section{Transients}

AMEGO's wide field of view, providing a regular all-sky survey as it sweeps across the sky every $\sim 90$ minutes makes it an ideal instrument for studying the time-variable sky. AMEGO will detect outbursts from many known source classes and likely from new source classes. In this proceedings, we focus on Gamma-ray Bursts, though AMEGO will also detect many classes of extragalactic (e.g. AGN, Supernovae), Galactic transients (e.g. Novae, Binaries, Pulsars, Magnetars), as well as possible counterparts to Fast Radio Bursts, Gravitational Wave Events, and high-energy Neutrinos.

\subsection{Gamma-ray Bursts (GRBs)}

GRBs are short-lived extremely energetic extragalactic transients resulting from both the mergers of neutron stars (or perhaps neutron stars with a black hole) and the collapse of massive stars. Both systems launch relativistic jets powered by a central black hole or rapidly spinning neutron star and produce gamma-ray emission lasting anywhere from a fraction of second to thousands of seconds, followed by longer-lived (hours-months) broadband (radio to high-energy gamma-ray) afterglows as the jet plows into the surrounding environment.

GRBs have been detected in the $\mathrm{MeV}$ band for decades, but mainly in background-dominated triggering instruments (e.g. CGRO-BATSE, Fermi-GBM, INTEGRAL SPI/ACS). These observations have led to many thousands of characterizations of the prompt emission temporal and spectral evolution, and localizations leading to follow-up afterglow observations across the electromagnetic spectrum from radio to high-energy $(\mathrm{GeV})$ gamma-rays. AMEGO will revolutionize GRBs once again by providing sensitive $\mathrm{MeV}$ observations of GRBs detecting both the short prompt emission as well as long-lived afterglows bridging regions of the broad spectra where the interplay of emission components is poorly understood.

Preliminary simulations of GRB detection rates, based upon the methods developed by [6], indicate that AMEGO will be a very prolific GRB detector (400+ per year, see Figure 3), including 10's per year at high redshifts. These GRB triggers will provide localizations for ground-based follow-up by wide field-of-view instruments, and characterization of $\mathrm{MeV}$ temporal and spectral evolution.

Most GRB prompt emission spectra have their peak energy in the AMEGO bandpass. AMEGO will provide detailed time-resolved spectra that will lead to understanding of emission mechanisms, and perhaps solve the debate over whether GRB jets are baryon or magnetically dominated. A handful of well observed GRB afterglows suggest that a single component produces the emission from radio to $\mathrm{GeV}$ energies, a challenge for Synchrotron shock models. AMEGO may be able to 
measure linear polarization of GRB prompt emission for a subset of bright GRBs. Sensitive observations in the $\mathrm{MeV}$ range will resolve if a modification to our understanding of the shock physics, or if a mechanism like magnetic reconnection is needed.
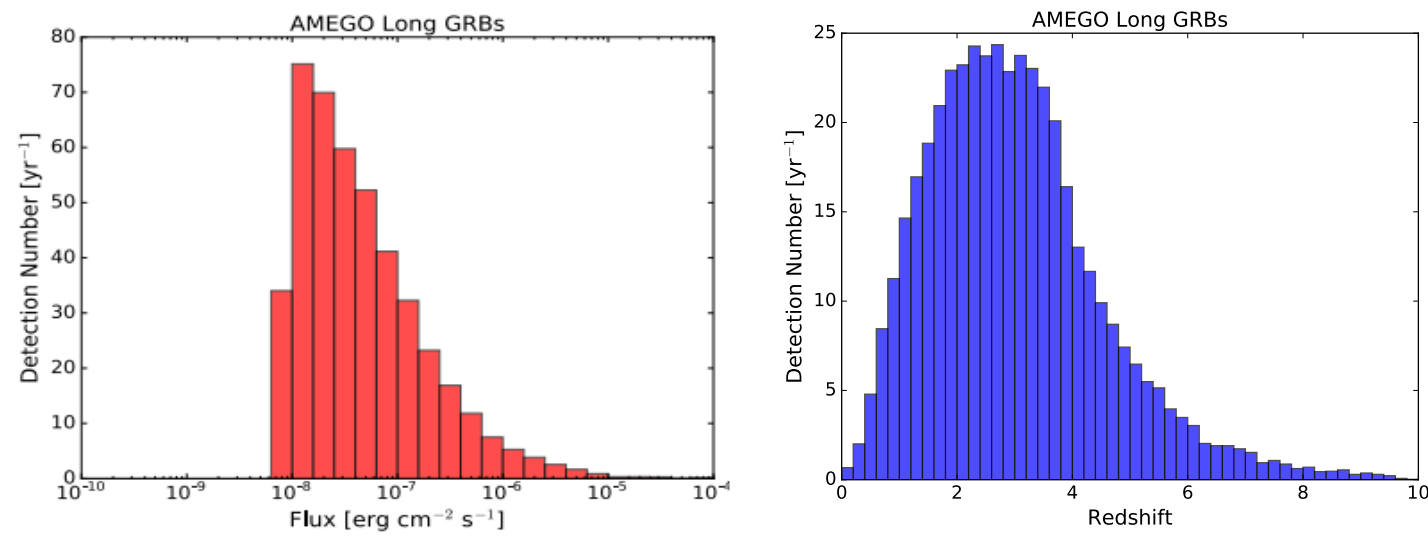

Figure 3: Predicted AMEGO GRB properties produced by applying a simplistic $100 \mathrm{~s}$ AMEGO sensitivity to a simulated intrinsic sample of GRBs [6]. The simulated GRB sample is scaled to Swift-BAT detected properties. The subset of GRBs above AMEGO's threshold and scaling to the AMEGO field of view provides the peak flux (left) and redshift (right) distributions for long GRBs.

\subsection{Fast Radio Bursts (FRBs)}

FRBs are relatively new source class [7], with no credible broadband counterparts and unknown progenitor. These short (millisecond) bursts of radio emission have now been detected from dozens of unique locations [8], with one object repeating many times [9]. The distances inferred from their dispersion measures put them distinctly of extragalactic origin. Although given the small fields of view of the discovery instruments, the intrinsic rate of these outbursts must be extremely high $\left(\sim 10^{4}\right.$ day $\left.^{-1}\right)$, their short duration makes them difficult to follow-up.

A wide range of theoretical models have been evoked to explain the observed properties of FRBs including pulsar giant flares [10], supramassive neutron stars [11] with possible short GRB association $[12,13]$, and many more exotic models. Although the broadband spectra of FRBs is not known, energetic phenomena that are bright in the radio (e.g. pulsars, AGN, etc.) tend to also be bright in the gamma-ray band. AMEGO's wide field of view and sensitivity make it an ideal instrument to search for counterparts to FRBs.

\section{Multi-Messenger Sources}

The fields of neutrino astronomy and gravitational wave astronomy have recently emerged with the discoveries of multiple extragalactic sources $[14,15]$. The origins of these messengers are of great interest to the astronomical community, but are difficult to discern given their transient nature and large localization regions. This will only be resolved with the detection of firmlyassociated broadband counterparts. AMEGO is well suited to detect these counterparts given the theoretical progenitor populations. 


\subsection{Gravitational Wave (GW) Counterparts}

AMEGO has important heritage in the search for GW counterparts, as demonstrated by Fermi Gamma-ray Burst Monitor (GBM) and Large Area Telescope (LAT) over the last couple of years $[16,17,18,19]$. The progenitors of short duration GRBs are thought to be the mergers of two neutron stars or a neutron star and black hole [20]. Therefore, it is widely accepted that GW triggers from LIGO/Virgo/KAGRA on these types of systems will be accompanied by a short gamma-ray burst if the jet is pointed at Earth. The GWs are slightly stronger along the rotation axis, enhancing the possibility of detection when a GRB is also present. The off-axis properties of short GRBs are more uncertain, but some models suggest potential off-axis gamma-ray emission [21].

The intriguing candidate signal associated with GW150914 detected by Fermi-GBM [16] also suggests the possibility of counterparts to binary black hole mergers, though additional signals from other black hole mergers are needed to confirm the association with GW150914-GBM.

AMEGO will provide all-sky coverage every 2 orbits ( $~ 90$ minutes), which lends itself very well to detecting GRB afterglows. Therefore even if the short GRB is not within the field of view at the time of the gravitational wave trigger, AMEGO is capable of detecting fading counterparts over the minutes-hours afterwards. Based upon observations by Fermi-GBM, AMEGO will likely be an extremely prolific detector of short GRBs, though detailed simulations have not yet been performed.

By the time AMEGO flies in the late-2020's, the ground-based GW network will have 35 participating interferometers (LIGO/Virgo/KAGRA), hopefully providing high uptime, and 3detector localizations for most events yielding sub-10's of $\mathrm{deg}^{2}$ localizations. Even with those localizations, wide field of view instruments are needed to better localize sources, and also to be available when only 2 detectors might see a particular source.

AMEGO GW counterparts would provide an independent confirmation of a signal (whether above threshold or elevating a sub-threshold signal). It would also probe the properties of the GRB signal including temporal and spectral evolution and energetics, and improved localization for follow-up observations with ground- and space-based telescopes, which in leads to detections of host galaxies and redshifts.

In addition to high-frequency GWs, Pulsar Timing Arrays (PTAs, [22]) and LISA [23] should also be detecting GWs by the late-2020's. Potential source classes for PTAs with electromagnetic counterparts including binary supermassive black holes with periods of order $\sim 1$ year [24], for which AMEGO's monitoring of blazars could find sources. LISA sources include merging supermassive black hole binaries and stellar mass binaries [25, 26], which could also potentially have gamma-ray counterparts.

\subsection{Neutrino Counterparts}

IceCube has detected dozens of neutrinos of astrophysical origin [14]. However, the sources of PeV neutrinos is unknown and an active area of study. Theoretically motivated options include Starburst galaxies, Blazars, high-fluence blazar flares with one potential association, optically thick sources, GRBs, Supernovae associated with low-luminosity GRBs, and choked-jet/Super Luminous Supernovae [27]. 
Broadband follow-up observations and archival searches have not yielded any firm electromagnetic counterparts. However, all of these source types have potential to be detectable in the $\mathrm{keV}$ to $\mathrm{MeV}$ band. AMEGO's superb sensitivity and wide field-of-view make it an ideal instrument to find an associated steady source at the location of the detected neutrinos, or detect coincident flaring during a neutrino detection. As $\mathrm{PeV}$ neutrinos continue to be collected by current and future facilities, the search will continue for electromagnetic counterparts. AMEGO is an ideal facility for this search and such a counterpart detection is needed to understand the origin of astrophysical neutrinos, which will also provide insight into the emission mechanisms at work in systems with extreme properties.

\section{Conclusions}

AMEGO will be an excellent mission to observe the transient Universe, and address some of the biggest outstanding problems in high-energy astrophysics.

\section{References}

[1] A. Moiseev, All-Sky Medium Energy Gamma-ray Observatory (AMEGO), in 35th International Cosmic Ray Conference (ICRC2017), vol. 35 of International Cosmic Ray Conference, p. 8, July, 2017.

[2] A. Moiseev, A. Bolotnikov, E. Hays, D. Thompson and R. James, High-Energy 3D position Resolution Calorimeter for the Use in Space Gamma-ray Astronomy based on position-sensitive virtual Frisch-grid CdZnTe detectors, in 35th International Cosmic Ray Conference (ICRC2017), vol. 35 of International Cosmic Ray Conference, p. 8, July, 2017.

[3] R. Caputo, F. Kislat and J. Racusin, AMEGO: Simulations of the Instrument Performance, in 35th International Cosmic Ray Conference (ICRC2017), vol. 35 of International Cosmic Ray Conference, p. 8, July, 2017.

[4] J. S. Perkins, M. Ajello, L. Marcotulli, E. Meyer, V. A. Paliya and T. Venters, All-Sky Medium Energy Gamma-ray Observatory (AMEGO), in 35th International Cosmic Ray Conference (ICRC2017), vol. 35 of International Cosmic Ray Conference, p. 8, July, 2017.

[5] R. Caputo, M. Meyer and M. A. Sánchez-Conde, AMEGO: Dark Matter, in 35th International Cosmic Ray Conference (ICRC2017), vol. 35 of International Cosmic Ray Conference, p. 8, July, 2017.

[6] A. Lien, T. Sakamoto, N. Gehrels, D. M. Palmer, S. D. Barthelmy, C. Graziani et al., Probing the Cosmic Gamma-Ray Burst Rate with Trigger Simulations of the Swift Burst Alert Telescope, ApJ 783 (Mar., 2014) 24, [1311.4567].

[7] D. R. Lorimer, M. Bailes, M. A. McLaughlin, D. J. Narkevic and F. Crawford, A Bright Millisecond Radio Burst of Extragalactic Origin, Science 318 (Nov., 2007) 777, [0 709.4301 ].

[8] D. Thornton, B. Stappers, M. Bailes, B. Barsdell, S. Bates, N. D. R. Bhat et al., A Population of Fast Radio Bursts at Cosmological Distances, Science 341 (July, 2013) 53-56, [1307 . 1628 ].

[9] L. G. Spitler, P. Scholz, J. W. T. Hessels, S. Bogdanov, A. Brazier, F. Camilo et al., A repeating fast radio burst, Nature 531 (Mar., 2016) 202-205, [1603.00581].

[10] M. Lyutikov, L. Burzawa and S. B. Popov, Fast radio bursts as giant pulses from young rapidly rotating pulsars, MNRAS 462 (Oct., 2016) 941-950, [1603.02891]. 
[11] H. Falcke and L. Rezzolla, Fast radio bursts: the last sign of supramassive neutron stars, A\&A 562 (Feb., 2014) A137, [1307.1409].

[12] B. Zhang, A Possible Connection between Fast Radio Bursts and Gamma-Ray Bursts, ApJL 780 (Jan., 2014) L21, [1310 . 4893].

[13] J.-S. Wang, Y.-P. Yang, X.-F. Wu, Z.-G. Dai and F.-Y. Wang, Fast Radio Bursts from the Inspiral of Double Neutron Stars, ApJL 822 (May, 2016) L7, [1603. 02014 ].

[14] M. G. Aartsen, M. Ackermann, J. Adams, J. A. Aguilar, M. Ahlers, M. Ahrens et al., Observation of High-Energy Astrophysical Neutrinos in Three Years of IceCube Data, Physical Review Letters 113 (Sept., 2014) 101101, [1405.5303].

[15] B. P. Abbott, R. Abbott, T. D. Abbott, F. Acernese, K. Ackley, C. Adams et al., GW170104: Observation of a 50-Solar-Mass Binary Black Hole Coalescence at Redshift 0.2, Physical Review Letters 118 (June, 2017) 221101, [1706.01812].

[16] V. Connaughton, E. Burns, A. Goldstein, L. Blackburn, M. S. Briggs, B.-B. Zhang et al., Fermi GBM Observations of LIGO Gravitational-wave Event GW150914, ApJL 826 (July, 2016) L6, [1602.03920].

[17] M. Ackermann, M. Ajello, A. Albert, B. Anderson, M. Arimoto, W. B. Atwood et al., Fermi-LAT Observations of the LIGO Event GW150914, ApJL 823 (May, 2016) L2, [1602 . 04488 ].

[18] J. L. Racusin, E. Burns, A. Goldstein, V. Connaughton, C. A. Wilson-Hodge, P. Jenke et al., Searching the Gamma-Ray Sky for Counterparts to Gravitational Wave Sources: Fermi GBM and LAT Observations of LVT151012 and GW151226, ApJ 835 (Jan., 2017) 82, [1606. 04901 ].

[19] O. b. o. t. Fermi-GBM and Fermi-LAT collaborations, Fermi observations of the LIGO event GW170104, ArXiv e-prints (June, 2017) , [1706.00199].

[20] R. Narayan, B. Paczynski and T. Piran, Gamma-ray bursts as the death throes of massive binary stars, ApJL 395 (Aug., 1992) L83-L86, [astro-ph/9204001].

[21] K. Hotokezaka, S. Wanajo, M. Tanaka, A. Bamba, Y. Terada and T. Piran, Radioactive decay products in neutron star merger ejecta: heating efficiency and $\gamma$-ray emission, MNRAS 459 (June, 2016) 35-43, [1511.05580].

[22] S. Burke-Spolaor, Gravitational-Wave Detection and Astrophysics with Pulsar Timing Arrays, ArXiv e-prints (Nov., 2015) , [1511.07869].

[23] A. Klein, E. Barausse, A. Sesana, A. Petiteau, E. Berti, S. Babak et al., Science with the space-based interferometer eLISA: Supermassive black hole binaries, PRD 93 (Jan., 2016) 024003, [1511.05581].

[24] M. Ackermann, M. Ajello, A. Albert, W. B. Atwood, L. Baldini, J. Ballet et al., Multiwavelength Evidence for Quasi-periodic Modulation in the Gamma-Ray Blazar PG 1553+113, ApJL 813 (Nov., 2015) L41, [1509.02063].

[25] P. Christian and A. Loeb, LISA detection of binary black holes in the Milky Way galaxy, MNRAS 469 (July, 2017) 930-937, [1701.01736].

[26] A. Sesana, Multi-band gravitational wave astronomy: science with joint space- and ground-based observations of black hole binaries, ArXiv e-prints (Feb., 2017) , [1702.04356].

[27] F. Halzen, High-energy neutrino astrophysics, Nature Physics 13 (Mar., 2017) 232-238. 